\title{
NuRenew - An Advanced Hybrid Nuclear-Renewable Energy Park
}

\author{
B. Petrovic \\ Georgia Institute of Technology \\ 770 State Street, Atlanta, GA 30332-0745, USA \\ Phone number: +1 (404) 789-9287, e-mail: bojan.petrovic@gatech.edu
}

\begin{abstract}
The paper will present a novel concept of a hybrid Nuclear-Renewable (NuRenew) energy park, originally introduced in 2011 [1-2], that promotes accelerated synergistic deployment of non-fossil power generating sources enabling economically viable phasing out of fossil power plants and their replacement by near-zero emission power sources (nuclear and renewables). While a number of other hybrid energy systems have been proposed [3], the NuRenew concept synergistically combines high-temperature liquid salt cooled reactors (LSCR), liquid salt based concentrated solar power (CSP), and liquid salt based thermal energy storage (TES), for the first time all tightly coupled together in a flexible, robust topology.
\end{abstract}

This integrated approach enables jointly developing high temperature salt technologies for all three systems, thus sharing the development costs, which otherwise would require an investment potentially too large for any individual technology by itself. Moreover, the energy storage is shared reducing the cost to each of the participating technologies. The Nu-Renew energy park is expected to include industries that use high process temperatures and significant amounts of energy, including coal-to-liquid, hydrogen production, water desalination, and manufacturing of PV-panels.

For the nuclear power plant, this energy park provides an opportunity to expand beyond the electricity production, to a range of high temperature process heat applications. To protect investment, some of these processes may require extremely high reliability and uninterrupted operation, which would not be possible to guarantee by the nuclear power itself without the thermal energy storage. For CSP, sharing of the TEST costs with nuclear may significantly enhance its economic viability. CSP offers great potential, but its mass deployment will not occur until its economic competitiveness is achieved and insolation intermittency is addressed. Deployment within the NuRenew will enable shared use (and thus cost reduction) of the common thermal energy storage to address the intermittency issue at an acceptable cost for CSP.

\section{Key words}

Hybrid energy park, process heat, high-temperature nuclear reactor, concentrated solar power, energy storage

\section{Challenges: Economics, Sustainability, Security, Safety, Waste}

Several significant challenges stem from the historical and current dependence on fossil fuels, and features of current low-carbon sources. According to the International Energy Agency [4], \$38 trillion is needed for new energy infrastructure worldwide. Importantly though, most countries, like the US, have significant resource and/or financial investments tied to coal, oil, gas, and their related infrastructure. They cannot afford to simply abandon these commitments. Current nuclear power plants with oncethrough uranium-based fuel cycle raise the issue of longterm sustainability both from the perspective of resources and ultimate nuclear waste management. At the same time, CSP with TES by itself still has a way to go to reach economic competitiveness. Production of electricity alone ( 1/3 of total energy consumption) by low-carbon sources is not sufficient. Clean energy alternatives need to expand into areas of transportation and industrial processes. Water scarcity for power plant cooling is also a growing problem. The system proposed here addresses all these challenges through an integrated approach that represents an economically, technologically, and socially acceptable pathway for transitioning from the presently unsustainable dependence on fossil fuels to an energy future that is based on a viable low carbon footprint alternative.

\section{Needs}

Aiming to extend low carbon sources to non-electricity application, the question becomes what is the range of temperatures needed to meaningfully provide process heat to high temperature processes (HTPs). Figure 1 depicts the ranges of operating temperatures required by various HTPs. While it is frequently assumed that the goal should be very high temperature, above $900 \mathrm{C}$, in fact many processes may be successfully accomplished with temperatures up to $700-800 \mathrm{C}$. 


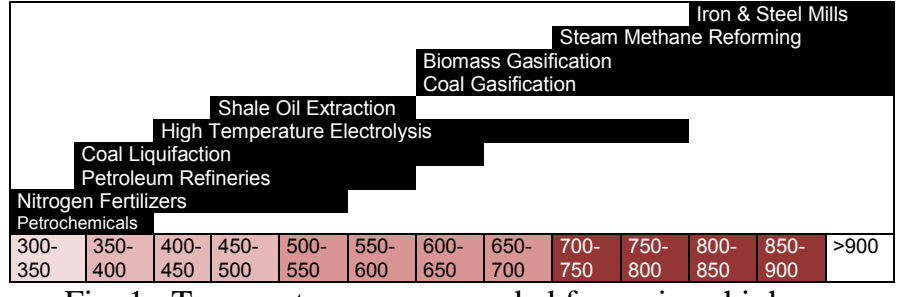

Fig. 1. Temperature ranges needed for various hightemperature processes.

Developing a system that delivers energy at higher temperature enables a broader range of applications, but also leads to increasing challenges related to the required HT materials. To quantify the impact of having certain temperature available, we used the EIA data [5] on the actual amount of process heat used by industry as a function of temperature. This is shown as the blue line in Figure 2. If we integrate the curve, and normalize by the total integral, we obtain the black line curve, which shows what percentage (fraction) of the total HTP needs could be covered by certain temperature, and thus the potential to expand beyond only electricity production.

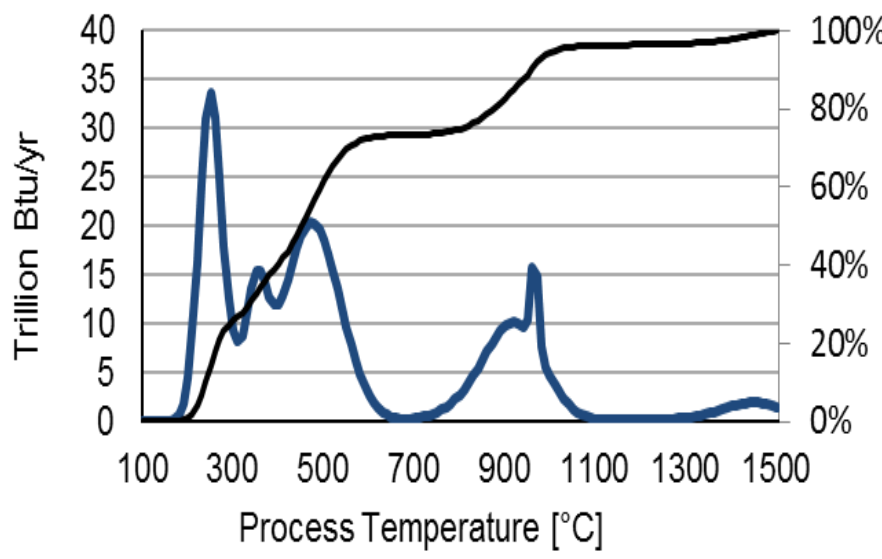

Fig. 2. Process heat use by temperature - differential and cumulative [based on EIA, 2009 data].

It turns out that very high temperatures are not needed to make a meaningful impact. For example, $700 \mathrm{C}$ will cover close to $80 \%$ HT energy needs, and increasing it to $850 \mathrm{C}$ or 900C will not significantly increase that percentage, while it will make the materials requirement significantly more challenging. Thus, while the target temperature remains to be determined, we are assuming that in the first stage the system will be designed to provide process heat at $\sim 700$ $750 \mathrm{C}$.

This decision is further supported when considering technology readiness of high-temperature reactor materials presented in Table I [6]. Essentially, for temperatures up to $\sim 700 \mathrm{C}$, "tested and approved" materials exist. Temperatures up to $\sim 850 \mathrm{C}$ would require some development and confirmatory testing, while a target temperature of $1,000 \mathrm{C}$ would require significant development efforts, both in terms of time and money needed to reach a commercial deployment stage.
Table I. Technology readiness of high temperature materials [6]

\begin{tabular}{|c|c|c|c|}
\hline System Element & @ $700^{\circ} \mathrm{C}$ & @ $850^{\circ} \mathrm{C}$ & @ $1000^{\circ} \mathrm{C}$ \\
\hline Graphite Internals & Toyo Tanso IG110 or 430 & Toyo Tanso IG110 or 430 & Toyo Tanso IG110 or 430 \\
\hline Reactor Vessel & Hastelloy-N & $\begin{array}{l}\text {-Ni-weld overlay on } 800 \mathrm{H} \\
\text {-Insulated low-alloy steel } \\
\text {-New Ni-based alloy }\end{array}$ & $\begin{array}{l}\text {-Interior-insulated low- } \\
\text { alloy steel }\end{array}$ \\
\hline $\begin{array}{l}\text { Core barrel \& } \\
\text { other internals }\end{array}$ & Hastelloy-N & $\begin{array}{l}\text {-C-C composite } \\
\text {-New Ni-based alloy }\end{array}$ & $\begin{array}{l}\text {-C-C composite } \\
\text {-SiC-SiC composite } \\
\text {-New refractory metal }\end{array}$ \\
\hline $\begin{array}{l}\text { Control rods and } \\
\text { internal drives }\end{array}$ & $\begin{array}{l}\cdot \text { C-C composites } \\
\cdot \text { Hastelloy-N } \\
\cdot \mathrm{Nb}-1 \mathrm{Zr}\end{array}$ & $\begin{array}{l}\cdot \mathrm{C}-\mathrm{C} \text { composites } \\
\cdot \mathrm{Nb}-1 \mathrm{Zr}\end{array}$ & $\begin{array}{l}\cdot \mathrm{C}-\mathrm{C} \text { composites } \\
\cdot \mathrm{Nb}-1 \mathrm{Zr}\end{array}$ \\
\hline IHX \& DRACS & Hastelloy-N & $\begin{array}{l}\text {-New Ni-based alloy } \\
\text {-Double-sided Ni cladding } \\
\text { on } 617 \text { or } 230\end{array}$ & $\begin{array}{l}\text {-C-C composite } \\
\text {-SiC-SiC composite } \\
\text {-Monolithic SiC }\end{array}$ \\
\hline $\begin{array}{l}\text { Secondary (salt- } \\
\text { to-gas) HX }\end{array}$ & $\begin{array}{l}\text { Coaxial extruded } 800 \mathrm{H} \\
\text { tubes with Ni-based } \\
\text { layer }\end{array}$ & $\begin{array}{l}\text {-New Ni-based alloy } \\
\text {-Coaxial extruded } 800 \mathrm{H} \\
\text { tubes with Ni-based layer }\end{array}$ & $?$ \\
\hline
\end{tabular}

Since electricity accounts for $\sim 1 / 3$ of the total primary energy use, extending new low-carbon-emitting generators to non-electricity end-uses could have a major positive impact on the environment. Additional analysis of the suitability of individual HTPs for integration within the energy park, accounting for the technology readiness of required HT materials, will enable selection of candidate HTPs and the target system temperature. The following HTPs would be viable candidates for the energy park at mid-high temperatures ( 700C): electricity generation; desalination, coal-to-liquid fuel, waste treatment with supercritical water oxidation, cement calcinations, and production of photovoltaic cells. Even the processes requiring higher temperatures, such as hydrogen production, iron smelting, and aluminum refining may be included by topping up the cycle and increasing the "midhigh" temperature ( 850C) with electrical or gas heating, or heat pumps, to $\sim 1,000 \mathrm{C}$ target temperature.

Also to be considered in the ultimate optimization of the system is the "bottoming cycle", cascading of working fluid temperatures for the various processes, starting with the highest temperature fluid going to the process that needs the highest temperature, followed by processes requiring lower temperatures.

\section{Proposed NeRenew Energy Park Concept}

NuRenew has a modular topology that synergistically combines concentrated solar power, nuclear power generation, and molten salt thermal energy storage together with industries that use high temperatures and/or significant amounts of energy for manufacturing (Figure 3 ). In addition to high-efficiency electricity production, optimally matched industries in the energy park (such as coal-to-liquid fuels production, water desalination, hydrogen production, smelting, waste water treatment, or photovoltaic panel production) efficiently utilize the total energy produced (both power and heat) by low-carbon sources thereby amplifying the positive environmental and economic impacts. 


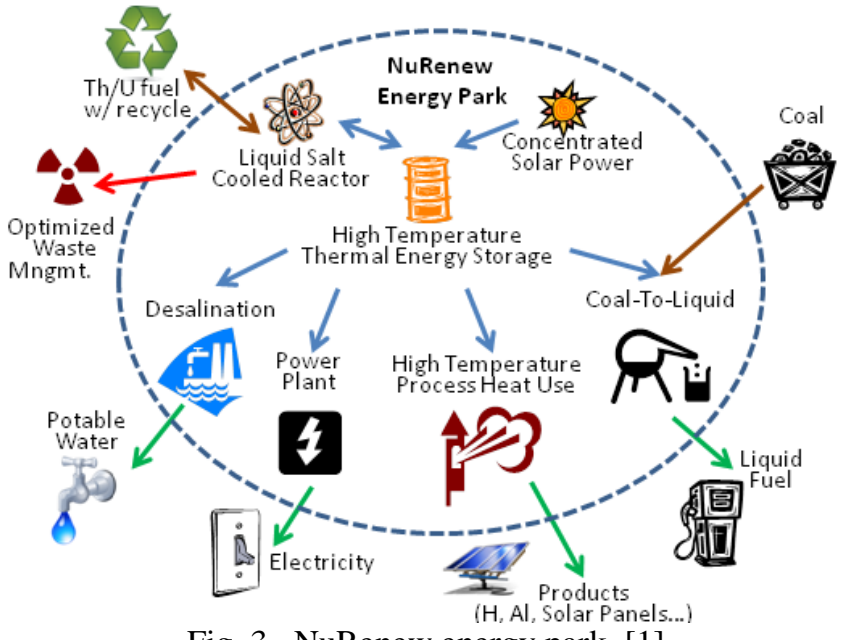

Fig. 3. NuRenew energy park. [1]

This concept offers several advantages. The modular topology of the power-generating components (CSP-TESLSCR) will be standardized to: 1) allow effective scaling; 2 ) enhance economics; and 3) enable adaptability to the regional environment by adjusting the configuration of the CSP-TES-LSCR components.

This integrated energy park concept provides the following attractive characteristics:

- Electricity generation

- Potable water generation

- High temperature process heat

- Compatible with coal-to-liquid and liquid fuel fabrication

- May provide energy for PV-cell manufacturing, thereby making the PV-cell lifetime cycle "greener"

- Inherently safe LSCR

- Compatible with the use of thorium fuel

- $\quad$ TES addresses intermittency of solar power (CSP)

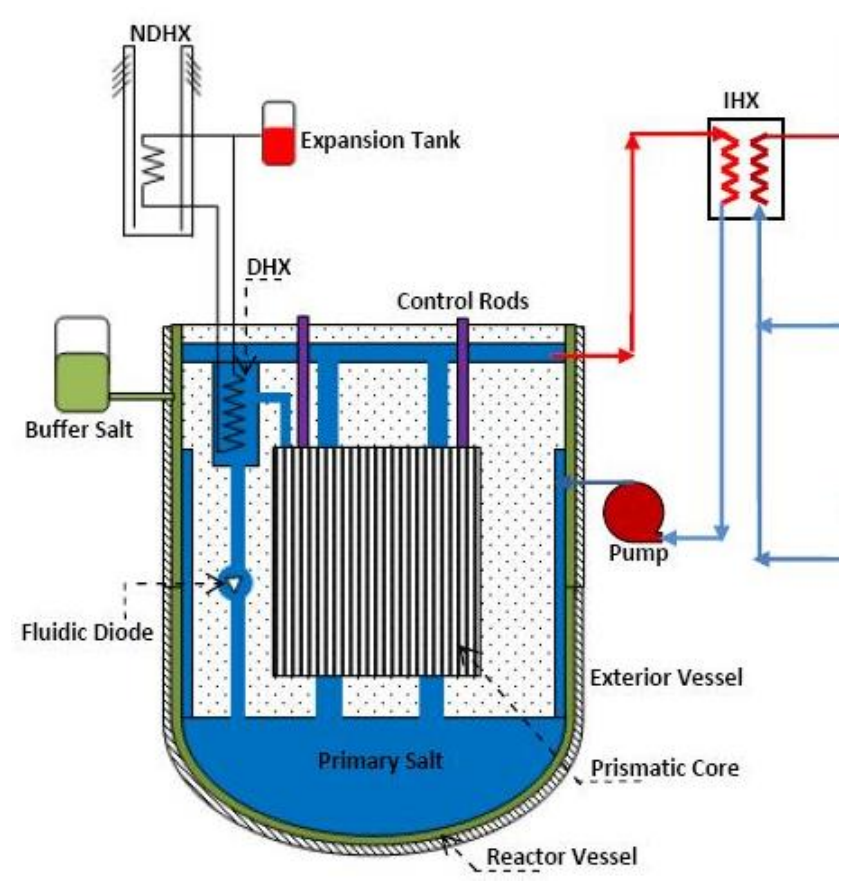

Fig. 4. NuRenew LSCR Schematic. [1]
On the nuclear power side, Liquid Salt Cooled Reactor (LSCR) is used (Figure 4). LSCR operates at low (slightly above atmospheric) pressure thus eliminating certain safety concerns of high pressure systems. It provides high temperature and consequently high efficiency (for energy generation), at the same time reducing requirements on cooling water. It implements inherent safety features addressing post-Fukushima concerns. Specific LSCR design envisioned for NuRenew will be based off the ORNL AHTR design [7].

CSP envisioned for NuRenew is based on the receiver in a tower concept and uses molten salt to achieve temperature comparable to that generated by LSCR.

Both nuclear-generated and solar-generated heat is transferred via respective primary heat exchangers from their primary loops to the secondary loop(s) that transfers and stores heat in a high temperature thermal energy storage, again based on using molten salt.

It is envisioned to implement the system with a robust topology, with modularity and multiple links between components to provide operational flexibility and stability, as illustrated in Fig. 5

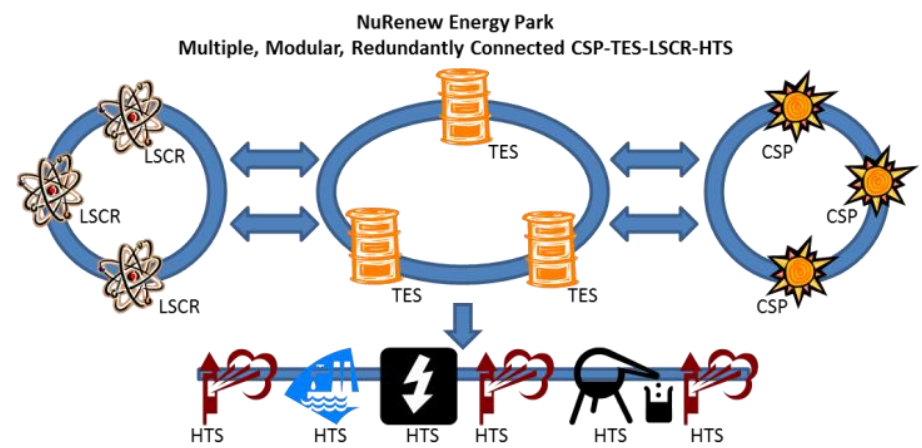

Fig. 5. NuRenew energy park - robust topology. [1]

Multiple LSCRs, multiple CSPs, and multiple TES provide redundancy and modularity. This reduces the impact when any single unit is off-line for maintenance or any other reason. At the same time, it allows modular construction, with staggered financing, making the overall project financially more viable. Multiple components are linked with multiple/redundant heat transfer connections, providing very high overall reliability of the system.

\section{Synergistic Concept and Economics}

Main challenge for CSP deployment, beside the capital cost of the CSP system itself (which is within the acceptable range), is in intermittency of solar radiation. This requires TES, which significantly increases the total capital cost and ultimately the cost of delivered energy (whether as electricity or as heat in case it is used for HTP rather than electricity production). 
Main challenges for NPP include:

To facilitate load follow when the grid so requires.

- To insure safety under all conceivable conditions and scenarios. Frequently, this boils down to having external power available to safely remove decay heat under off-normal conditions.

Both of these challenges may again be addressed by TES. NPP requires only a moderately-sized TES, with energy storage capacity on the order of a few full-power hours, significantly less than the storage required by CSP, if both are normalized to unit nominal full power. This proportionally smaller storage of NPP would not impact its economics as significantly as the CSP TES does, but it would be significant enough that a TES cost saving is highly desirable.

The synergy in economics aspect comes from the fact that, if LSCR and CSP are integrated via a common, combined TES for both LSCR and CSP, it does not have to be in size as large as the sum of the two individual TES. The reason is that individual sizing is not deterministic, but based on the probabilistic assessment. Since the two systems have different storage requirements characteristics (given by individual requirement probability density functions), a less-than-combined-size common storage may provide the same level of reliability (effectiveness) as the two individual ones. With proper sizing, having the ratio of nuclear and solar system power approximately equal to the ratio of the corresponding individual TES capacity, this synergy may be effectively utilized. More detailed studies are planned to optimize the sizing based on actual representative production and consumption time-profiles.

As an example, consider for simplicity a system with a nominal peak powers of $250 \mathrm{MW}$ solar and 1,000 MW nuclear, together with a target of 16 hrs energy storage for solar, and $4 \mathrm{hrs}$ for nuclear. That would translate into required capacity of $1 \mathrm{GW}$-hr TES each for solar and nuclear. However, when combined, preliminary estimates suggest that $\sim 1.4 \mathrm{GW}-\mathrm{hr}$ TES would provide a similar functionality. If the corresponding cost is split evenly, it would result in a 30\% TES cost saving for each system. Moreover, a single larger TES would likely be somewhat cheaper than two individual systems, further increasing the savings. The multi-purpose sharing of TES in NewRenew reduces its effective cost significantly, enabling the earlier competitive deployment of CSP systems, increasing energy supply, and creating a reliable, low-carbon supply of electricity. Additionally, the cost of high-temperature molten salt technology development is also shared between the solar and nuclear portions of the system.

\section{Conclusions}

The NuRenew hybrid energy park concept provides a pathway for accelerated deployment of CSP, in conjunction with low-emitting nuclear power, and transitioning from the current carbon based energy paradigm to a sustainable energy future. It supports transition industries such as coalto-liquid (CTL) and PV-cell manufacturing. While not carbon-free by itself, CTL permits economically-acceptable accelerated phasing out of fossil-fired power plants, while enabling continued use of large US coal resources and infrastructure. This will immediately improve energy security as well as reduce the environmental impact of transportation fuels. Later, if carbon capture and sequestration becomes viable, its addition could make CTL a zero carbon option. Moreover, clean electricity and high temperature process heat generated by NewRenew would enable environmentally friendly production of PVcells, hydrogen, potable water.

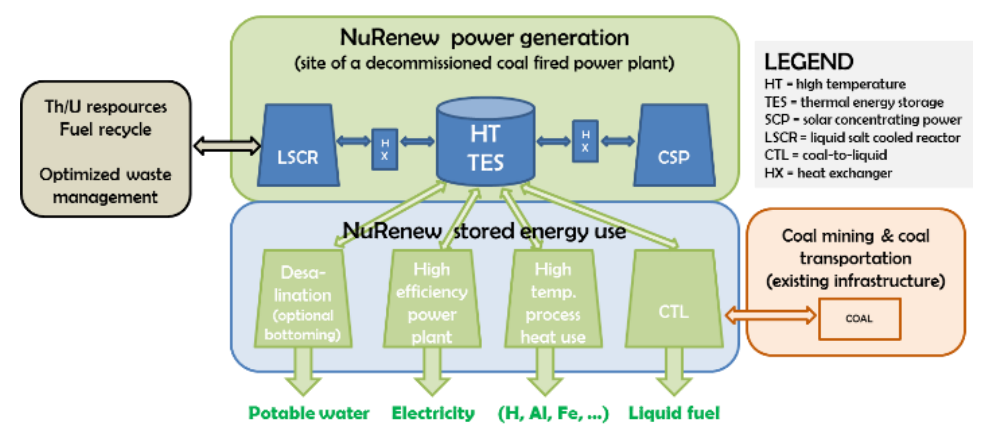

Fig. 6. NuRenew energy park concept

\section{References}

[1] B. Petrovic, M. Begovic, M. Brown, J. Crittenden, J.C. Santamarina, Z. Ma (NREL), "NuclearRenewable Energy Park NuRenew," Proposal to NSF, 2011.

[2] B. Petrovic, "NuRenew: Hybrid Nuclear (LSCR)Renewable(CSP) Energy Park," presented at the Workshop on Hybrid Energy Systems, Salt Lake City, UT, April 3-4, 2012 (HES-2012).

[3] M. Green, P. Sabharwall, M. Mckellar, Su-Jong Yoon, C. Abel, B. Petrovic, D. Curtis, "Nuclear Hybrid Energy System: Molten Salt Energy Storage," Report INL/EXT-13-31768, Idaho National Laboratory (November 2013).

[4] International Energy Agency (IEA), World Energy Outlook 2011, Paris, 660 pages, 2011.

[5] Energy Information Administration; The national energy modeling system: An overview 2009.

[6] B. Corwin, Fluoride Salt Cooled High Temperature Reactor (FHR) Materials Challenges. Presentation at the ORNL FHR workshop, Sep. 20, 2010, https://www.ornl.gov/fhr/presentations/Corwin.pdf.

[7] D. E. Holcomb, F. J. Peretz, A. L. Qualls, "Advanced High Temperature Reactor Systems and Economic Analysis," ORNL/TM-2011/364, Oak Ridge National Laboratory, Oak Ridge, Tenn. (September 2011). 